\title{
Verrucous Carcinoma in a Wounded Military Amputee
}

\author{
Kelly Laskoski, MD; Danielle Neal, DO; Brittany Lenz, MD; Margaret Abuzeid, MD; \\ Thomas Beachkofsky, MD
}

\section{PRACTICE POINTS}

- Verrucous carcinoma is a rare, well-differentiated, locally aggressive squamous cell carcinoma that commonly occurs in sites of inflammation or chronic irritation.

- Histologically, verrucous carcinoma can be mistaken for other entities including verruca vulgaris, keratoacanthoma, and pseudoepitheliomatous hyperplasia, often delaying the appropriate diagnosis and treatment.

To the Editor:

Verrucous carcinoma is a rare, well-differentiated, locally aggressive squamous cell carcinoma first described by Ackerman in 1948. ${ }^{1}$ There are 4 main clinicopathologic types: oral florid papillomatosis or Ackerman tumor, giant condyloma acuminatum or Buschke-Lowenstein tumor, plantar verrucous carcinoma, and cutaneous verrucous carcinoma. ${ }^{2,3}$ Historically, most patients are older white men. The lesion commonly occurs in sites of inflammation ${ }^{4}$ or chronic irritation/trauma. Clinically, patients present with a slowly enlarging, exophytic, verrucous plaque violating the skin, fascia, and occasionally bone. Although these lesions have little tendency to metastasize, substantial morbidity can be seen due to local invasion. Despite surgical excision, recurrence is not uncommon and is associated with a poor prognosis and higher infiltrative potential. ${ }^{5}$

A 45-year-old male veteran initially presented to our dermatology clinic with a $4-\mathrm{cm}$, macerated, verrucous plaque on the left lateral ankle in the area of a skin graft placed during a prior limb salvage surgery (Figure 1). The patient experienced a traumatic blast injury while deployed 7 years prior with a subsequent right-sided below-the-knee amputation and left lower limb salvage. The lesion was clinically diagnosed as verruca vulgaris and treated with daily salicylic acid. Six weeks after the initial presentation, the lesion remained largely unchanged. A biopsy subsequently was obtained to confirm the diagnosis. At that time, the histopathology was consistent with verruca vulgaris without evidence of carcinoma. Due to the persistence of the lesion, lack of improvement with topical treatment, and overall size, the patient opted for surgical excision.

A year later, the lesion was excised again by orthopedic surgery, and the tissue was submitted for histopathologic evaluation, which was suggestive of a verrucous neoplasm with some disagreement on whether it was consistent with verrucous hyperplasia or verrucous carcinoma. Following excision, the patient sustained a nonhealing chronic ulcer that required wound care for a total of 6 months. The lesion recurred a year later and was surgically excised a third time. A split-thickness skin graft was utilized to repair the defect. Histopathology again was consistent with verrucous carcinoma. With a fourth and final recurrence of the verrucous plaque 6 months later, the patient elected to undergo a left-sided belowthe-knee amputation.

Verrucous carcinoma can represent a diagnostic dilemma, as histologic sections may mimic benign entities. The features of a well-differentiated squamous epithelium

From Wilford Hall Ambulatory Surgical Center, Joint Base San Antonio-Lackland AFB, Texas. Drs. Laskoski, Neal, Lenz, and Beachkofsky are from the Dermatology Department. Dr. Abuzeid is from the Pathology Department.

The authors report no conflict of interest.

The view expressed herein do not reflect the official policy or position of the Department of the Air Force, Department of the Army, or the US Government. Correspondence: Kelly Laskoski, MD, 11914 Alydar Loop, Colorado Springs, CO 80921 (kelly.c.laskoski.mil@mail.mil).

doi:10.12788/cutis.0332 

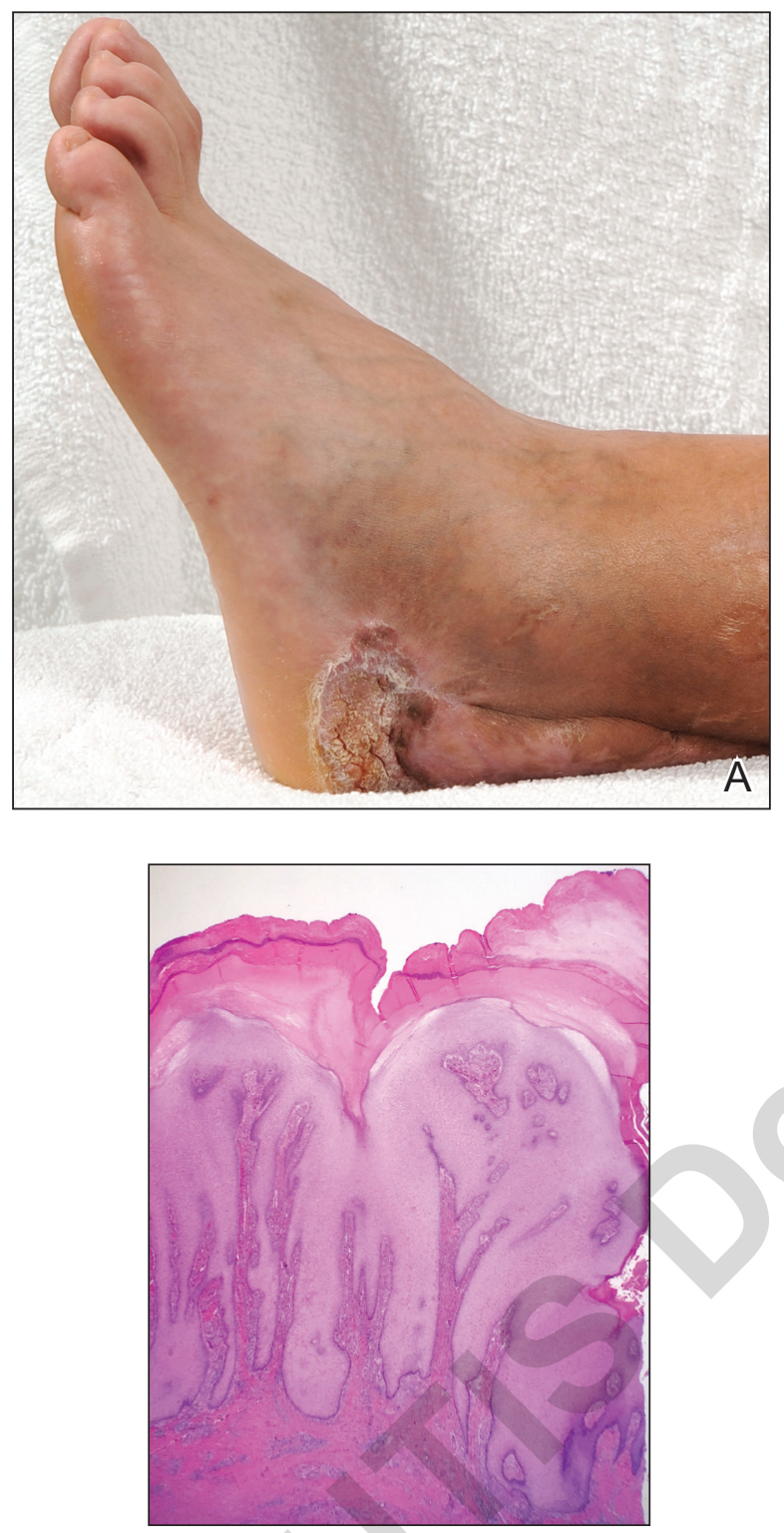

FIGURE 2. Biopsy of the lesion demonstrated minimal squamous atypia in a predominantly exophytic squamous proliferation, with focal endophytic pushing borders by rounded bulbous rete ridges into the mid and deep dermis (H\&E, original magnification $\times 40$ ).

with hyperkeratosis, papillomatosis, and acanthosis can be mistaken for verruca vulgaris, keratoacanthoma, and pseudoepitheliomatous hyperplasia, ${ }^{6}$ which are characteristic of verrucous hyperplasia. Accurate diagnosis can be difficult with a superficial biopsy because of the mature appearance of the epithelium, ${ }^{7}$ prompting the need for multiple and deeper biopsies ${ }^{8}$ to include sampling of the base of the hyperplastic epithelium in which the characteristic bulbous pushing growth pattern of the rete ridges is visualized. Precise histologic diagnosis can be further confounded by external mechanical factors, such as pressure, which can distort the classic histopathology. ${ }^{7}$ The histopathologic features leading to the diagnosis

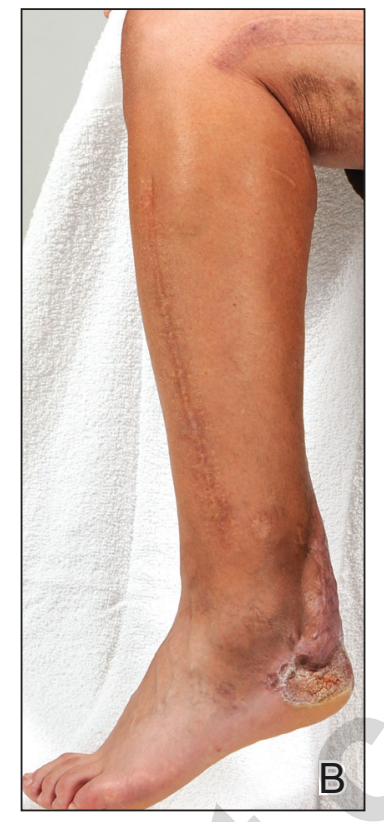

FIGURE 1. Verrucous carcinoma. A, A large, exophytic, verrucous plaque on the left lateral ankle in an area of prior skin graft placement. B, Multiple adjacent surgical scars from prior limb salvage surgery.

of verrucous carcinoma in our specimen were minimal squamous atypia present in a predominantly exophytic squamous proliferation with human papillomavirus cytopathic effect and focal endophytic pushing borders by rounded bulbous rete ridges into the mid and deep dermis (Figure 2).

Diagnostic uncertainty can delay surgical excision and lead to progression of verrucous carcinoma. Unfortunately, even with appropriate surgical intervention, recurrence has been documented; therefore, close clinical follow-up is recommended. The tumor spreads by local invasion and may follow the path of least resistance. ${ }^{4}$ In our patient, the frequent tissue manipulation may have facilitated aggressive infiltration of the tumor, ultimately resulting in the loss of his remaining leg. Therefore, it is important for clinicians to recognize that verrucous carcinoma, especially one that develops on a refractory ulcer or scar tissue, may be a complex malignant neoplasm that requires extensive treatment at onset to prevent the amputation of a limb.

\section{REFERENCES}

1. Ackerman LV. Verrucous carcinoma of the oral cavity. Surgery. 1948;23:670-678.

2. Yoshitasu S, Takagi T, Ohata C, et al. Plantar verrucous carcinoma: report of a case treated with Boyd amputation followed by reconstruction with a free forearm flap. J Dermatol. 2001;28:226-230.

3. Schwartz R. Verrucous carcinoma of the skin and mucosa. J Am Acad Dermatol. 1995;32:1-14.

4. Bernstein SC, Lim KK, Brodland DG, et al. The many faces of squamous cell carcinoma. Dermatol Surg. 1996;22:243-254.

5. Costache M, Tatiana D, Mitrache L, et al. Cutaneous verrucous carcinoma-report of three cases with review of literature. Rom J Morphol Embryol. 2014;55:383-388.

6. Shenoy A, Waghmare R, KavishwarV, et al. Carcinoma cuniculatum of foot. Foot. 2011;21:207-208.

7. Klima M, Kurtis B, Jordan P. Verrucous carcinoma of skin. J Cutan Pathol.1980;7:88-98.

8. Pleat J, Sacks L, Rigby H. Cutaneous verrucous carcinoma. Br J Plast Surg. 2001;54:554-555. 